\title{
QUANTITATIVE EVALUATIONS OF THE CONTRIBUTION OF THE EXCITATORY IONIC CONDUCTANCE TO REPETITIVE SPIKING IN A MATHEMATICAL MODEL OF MEDIAL VESTIBULAR NUCLEUS NEURONS
}

\author{
SHORT COMMUNICATION \\ TAKAAKI SHIRAHATA* \\ Laboratory of Pharmaceutical Education, Kagawa School of Pharmaceutical Sciences, Tokushima Bunri \\ University, 1314-1 Shido, Sanuki, Kagawa 769-2193, Japan
}

(Received: August 24, 2015; accepted: October 28, 2015)

\begin{abstract}
Medial vestibular nucleus neurons show spontaneous repetitive spiking. This spiking activity was reproduced by a Hodgkin-Huxley-type mathematical model, which was developed in a previous study. The present study performed computer simulations of this model to evaluate the contribution of the excitatory ionic conductance to repetitive spiking. The present results revealed the difference in the influence of the transient sodium, persistent sodium, and calcium conductance on spiking activity. The differences between the present and previous results obtained from other neuronal mathematical models were discussed.
\end{abstract}

Keywords: Medial vestibular nucleus neurons - spiking - mathematical model -sodium conductance calcium conductance

The Hodgkin-Huxley model is an important mathematical model in the field of neuroscience [2]. Analyses of various neuron models based on the Hodgkin-Huxley model are very useful for understanding the characteristics of ionic conductance [3-8]. Previous studies have revealed that repetitive spiking of various neurons is generated by an interaction between persistent and transient sodium conductance [4-6] or between calcium and sodium conductance $[3,7]$. The present study investigated the Hodgkin-Huxley-type mathematical model, which describes spontaneous repetitive spiking of type B medial vestibular nucleus neurons (MVNn) [1]. MVNn are second-order neurons within the vestibulo-ocular and vestibulo-spinal network, and play an important role for computation of internal representations of head movement velocity in the horizontal plane [1]. This model includes the persistent sodium, transient sodium, and calcium conductance. However, the contribution of these three ionic conductance to spiking was not clarified in a previous report [1]. Therefore, in the present study, we performed computer simulation analysis of the type B MVNn

\footnotetext{
*E-mail address: tshi@kph.bunri-u.ac.jp
} 


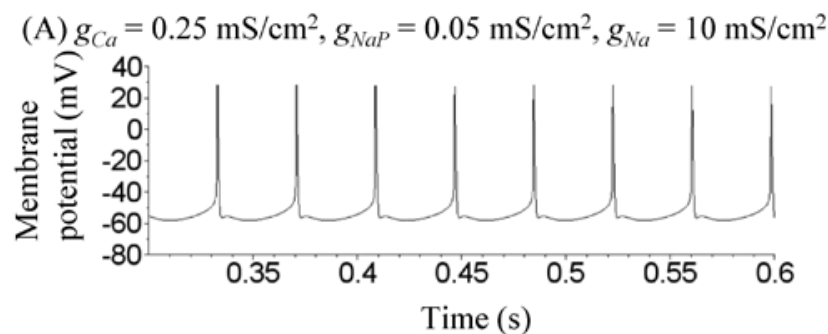

(B) $g_{C a}=0.00 \mathrm{mS} / \mathrm{cm}^{2}, g_{N a P}=0.05 \mathrm{mS} / \mathrm{cm}^{2}, g_{N a}=10 \mathrm{mS} / \mathrm{cm}^{2}$

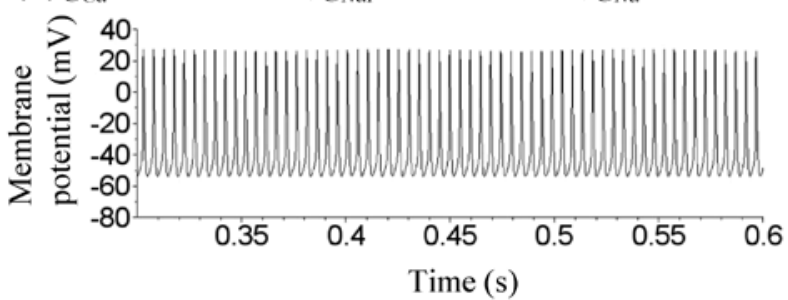

(C) $g_{C a}=0.25 \mathrm{mS} / \mathrm{cm}^{2}, g_{N a P}=0.00 \mathrm{mS} / \mathrm{cm}^{2}, g_{N a}=10 \mathrm{mS} / \mathrm{cm}^{2}$

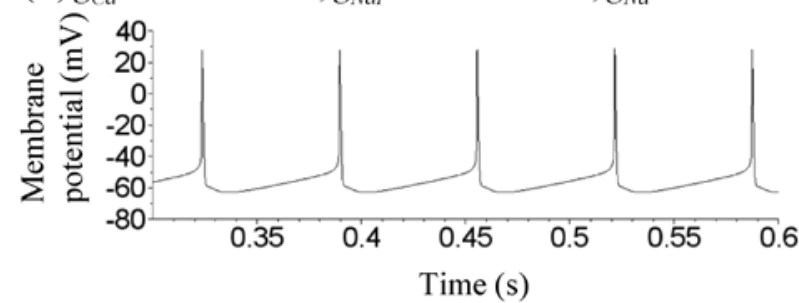

(D) $g_{C a}=0.25 \mathrm{mS} / \mathrm{cm}^{2}, g_{N a P}=0.05 \mathrm{mS} / \mathrm{cm}^{2}, g_{N a}=0 \mathrm{mS} / \mathrm{cm}^{2}$

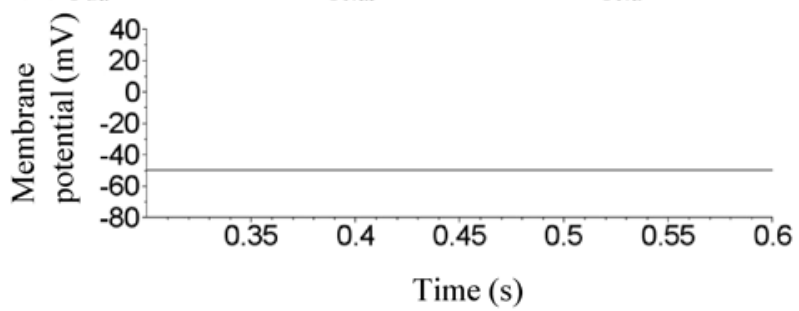

Fig. 1. Time courses of the simulated membrane potential. (A) $g_{C a}, g_{N a P}$, and $g_{N a}=0.25,0.05$, and 10 , respectively. (B) $g_{C a}, g_{N a P}$, and $g_{N a}=0.00,0.05$, and 10 , respectively. (C) $g_{C a}, g_{N a P}$, and $g_{N a}=0.25,0.00$, and 10 , respectively. (D) $g_{C a}, g_{N a P}$, and $g_{N a}=0.25,0.05$, and 0 , respectively 
model and aimed to reveal the relationship between spiking and these three conductance in detail.

The model analyzed in the present study was previously developed [1] and is described by a system of coupled nonlinear ordinary differential equations (ODEs) (Model equations are provided in " 2 Model development" in [1]). In order to investigate the relationship between spiking and the three conductance, the present study focused on three problem parameters in the ODEs, the maximal conductance of persistent sodium current $\left(g_{N a P}\right)$, transient sodium current $\left(g_{N a}\right)$, and calcium current $\left(g_{C a}\right)$, and systematically varied these parameter values to obtain numerical results of the ODEs. Numerical results were obtained using the free and open source software Scilab (http://www.scilab.org/).

Figure 1 shows the time courses of the membrane potentials of the model under different conditions. When $g_{C a}, g_{N a P}$, and $g_{N a}$ were set at default values (Fig. 1A), the model showed spontaneous repetitive spiking as previously reported (Fig. 2a in [1]). When $g_{C a}$ was blocked, the model showed spiking, but the spiking frequency was greater than that in Figure 1A (Fig. 1B). When $g_{N a P}$ was blocked, the model showed spiking, but the frequency was smaller than that in Figure 1A (Fig. 1C). When $g_{N a}$ was blocked, spiking was completely blocked (Fig. 1D).

The dynamic states of the model in the $\left(g_{\mathrm{Ca}}, g_{\mathrm{Na}}\right)$-parameter space under different $g_{N a P}$ conditions (Figs $2 \mathrm{~A} 1,2 \mathrm{~A} 2$, and 2A3) and in the $\left(g_{N a P}, g_{N a}\right)$-parameter space under different $g_{C a}$ conditions (Figs 2B1, 2B2, and 2B3) were revealed. Irrespective of $g_{\text {NaP }}$ values, the $\left(g_{C a}, g_{N a}\right)$-parameter space consisted of a spontaneous repetitive spiking state (black circle) and a quiescent state (white circle), and a decrease in $g_{\mathrm{Ca}}$ induced a decrease in the $g_{\mathrm{Na}}$ threshold required to induce spiking. Irrespective of $g_{\mathrm{Ca}}$ values, the $\left(g_{\mathrm{NaP}}, g_{\mathrm{Na}}\right)$-parameter space consisted of a spontaneous repetitive spiking state (black circle) and a quiescent state (white circle), and a decrease in $g_{N a P}$ induced an increase in the $g_{N a}$ threshold required to induce spiking.

Three important conclusions can be drawn based on the above results. First, although a previous study [1] did not indicate the difference in the contribution of $g_{N a P}, g_{N a}$, and $g_{C a}$ on spiking, the present study revealed that $g_{N a}$ was indispensable, whereas both $g_{N a P}$ and $g_{C a}$ were dispensable to spiking under a default condition (Fig. 1). Therefore, it is considered that the ionic channel mediating the transient sodium current plays a more important role in generating spontaneous repetitive spiking of type B MVNn than the ionic channels mediating the persistent sodium and calcium currents. Second, the present study revealed the relationship between spiking and the sodium conductance. Previous studies indicated that a decrease in the persistent sodium conductance induced either an increase in the transient sodium conductance threshold required to induce spiking (Type 1) (Fig. 2 in $[4,6]$ ) or a decrease in the transient sodium conductance threshold (Type 2) (Fig. 2 in [5]). The results of the present study (Fig. 2B) indicate that the MVNn model can be categorized into Type 1 (see above). Third, the present study discovered a novel type of relationship between spiking and the calcium and sodium conductance. Previous studies indicated that a decrease in the calcium conductance induced an increase in the sodium conductance threshold required to induce spiking (Fig. 4 in [3] and Fig. 2 in [7]). In 
contrast, the results of the present study indicate that a decrease in the calcium conductance induced a decrease in the sodium conductance threshold required to induce spiking (Fig. 2A). Generally speaking, activation of the calcium channel leads neurons to a more excitable state. However, surprisingly, it is conjectured that the calcium channel of type B MVNn has a suppressive effect on the repetitive spiking. The present numerical results are predictions of the electrophysiological recording of type B MVNn spiking behavior in in vitro under various strengths of the sodium and calcium conductance. The present numerical predictions will be tested experimentally by manipulating the conductance strengths by dynamic clamp technique.

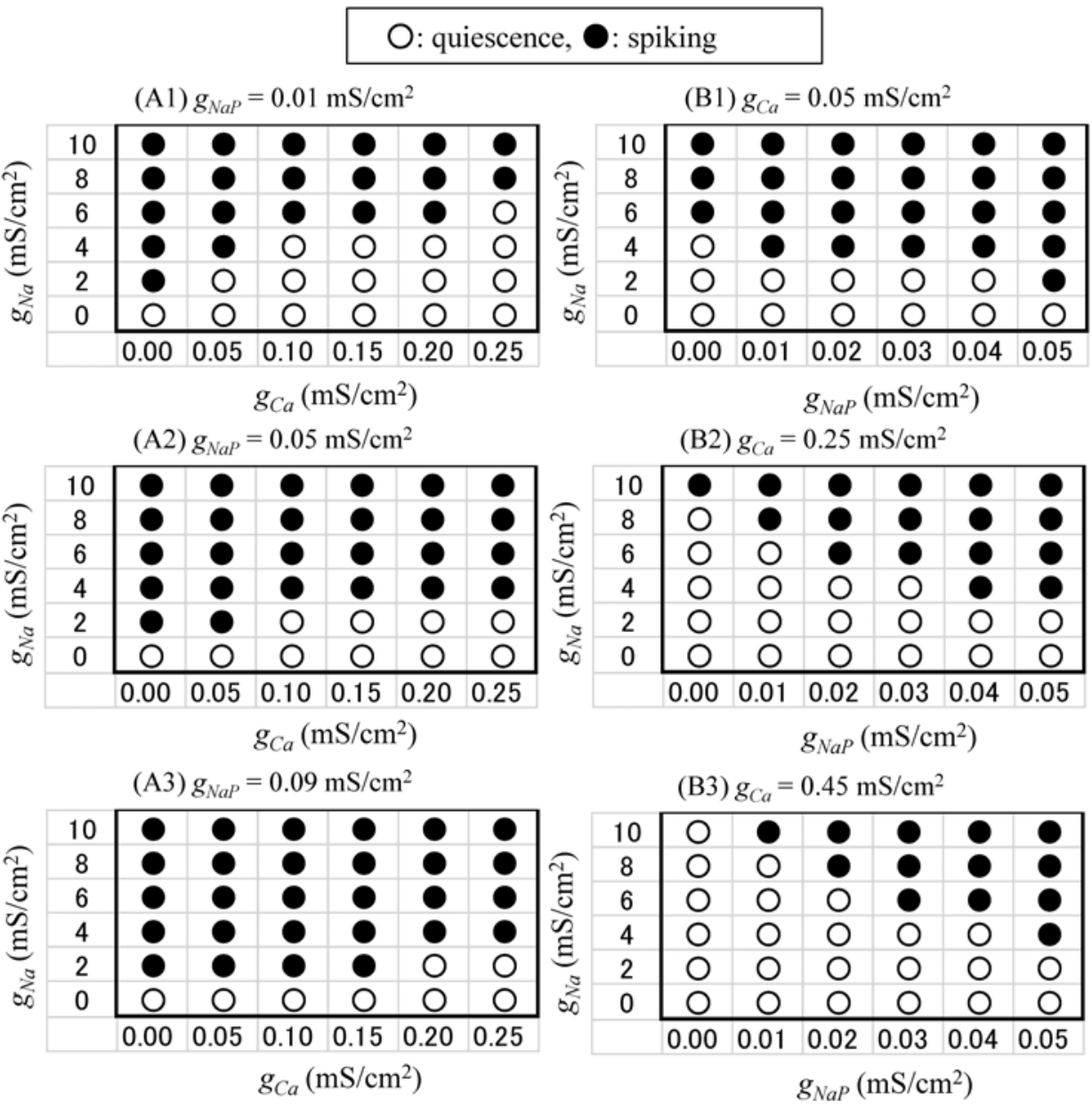

Fig. 2. State diagrams of the model. (A1), (A2), and (A3), The $\left(g_{C a}, g_{N a}\right)$-parameter space under different $g_{N a P}$ conditions. (B1), (B2), and (B3), The $\left(g_{N a P}, g_{N a}\right)$-parameter space under different $g_{C a}$ conditions. Black circle indicates a spontaneous repetitive spiking state, whereas white circle indicates a quiescent state 
ACKNOWLEDGEMENT

The author would like to thank Enago (www.enago.jp) for the English language review.

\section{REFERENCES}

1. Av-Ron, E., Vidal, P. P. (1999) Intrinsic membrane properties and dynamics of medial vestibular neurons: a simulation. Biol. Cyber. 80, 383-392.

2. Catterall, W. A., Raman, I. M., Robinson, H. P. C., Sejnowski, T. J., Paulsen, O. (2012) The HodgkinHuxley heritage: from channels to circuits. J. Neurosci. 32, 14064-14073.

3. Drion, G., Massotte, L., Sepulchre, R., Seutin, V. (2011) How modeling can reconcile apparently discrepant experimental results: The case of pacemaking in dopaminergic neurons. PLoS Comput. Biol. 7, e1002050.

4. Shirahata, T. (2011) The effect of variations in sodium conductance on pacemaking in a dopaminergic retinal neuron model. Acta Biol. Hung. 62, 211-214.

5. Shirahata, T. (2014) Effect of sodium conductance variations on electrical behavior of a neocortical neuron model. Acta Biol. Hung. 65, 379-384.

6. Shirahata, T. (2015) Numerical study of a mathematical model of vibrissa motoneurons: The relationship between repetitive spiking and two types of sodium conductance. Int. J. Theor. Math. Phys. 5, $48-52$.

7. Shirahata, T. (2015) Numerical simulation analysis of a mathematical model of circadian pacemaker neurons. Appl. Math. 6, 1214-1219.

8. Szücs, A. Huerta, R. (2015) Differential effects of static and dynamic inputs on neuronal excitability. J. Neurophysiol. 113, 232-243. 